\title{
Three Addend Addition: Who Goes Out of Order and Why?
}

\author{
Madhur Sharma $^{a}$, Satwat Bashir ${ }^{\mathrm{b}}$, Gaurav Suri ${ }^{\mathrm{c}}$ \\ [a] Department of Cognitive Science, University of California at Berkeley, Berkeley, CA, USA. [b] Department of Psychology, Stanford University, Stanford, CA, \\ USA. [c] Department of Psychology, San Francisco State University, San Francisco, CA, USA.
}

Journal of Numerical Cognition, 2021, Vol. 7(1), 66-81, https://doi.org/10.5964/jnc.6169

Received: 2020-09-18 • Accepted: 2021-02-03 • Published (VoR): 2021-03-31

Corresponding Author: Gaurav Suri, Department of Psychology, San Francisco State University, 1600 Holloway Ave, San Francisco, CA 94132, USA. E-mail: suri@sfsu.edu

Supplementary Materials: Materials [see Index of Supplementary Materials]

\begin{abstract}
Single-digit, three addend sums of the type $a+b+c$ offer a rich opportunity to directly observe the range of strategies that different participants may use because they afford the possibility of measuring a partial sum (i.e., $a+b$ or $a+c$ or $b+c$ ). For example, while computing the sum $9+7+1$, do participants go in order by first adding $9+7$ and then adding 1 , or do they incur the cost of going out of order by adding $9+1$ in order to obtain the partial sum of 10 , which makes the subsequent addition of 7 less effortful? Informed by findings in simple and complex arithmetic, we investigated the problem types and participant characteristics that can predict out of order switching behavior in such three-addend sums. To test our hypotheses, we tasked participants, first in an online study, and then in an in-person study to complete 120 single-digit, three addend problems. We found that participants switched the order of addition to prioritize efficiency gains in contexts in which the partial sum addends were small or equal to each other, or when doing so led to a partial sum of 10 , or led to a partial sum that is equal to the third remaining integer. Response latency data confirmed that participants were deriving efficiencies in the manner we expected. Related to individual differences, our findings showed that participants with higher levels of math education were most likely to seek efficiency benefits whenever they were on offer.
\end{abstract}

\section{Keywords}

mathematical cognition, addition strategies, cognitive effort, mathematical education

Imagine being asked to perform the following addition involving three single-digit numbers: $9+7+1$. How might you proceed? One approach is to advance from left to right and add $9+7$ to get a partial sum of 16 , and then to add 1 to get 17 as the final answer. Another approach is to notice that the first and third addend $(9+1)$ total to the partial sum of 10 which simplifies the subsequent addition of 7 to get the final answer of 17 . The latter approach relies upon the greater ease of adding any number to 10 and produces a more efficient overall process - even though it involves the initial cognitive cost of summing the addends out of order (the efficiency gains are even more tangible in sums of the type $97+82+3$ ). In this work, we seek to investigate the mathematical strategies people use to solve such three addend sums.

A large prior literature (see Campbell, 2005 for an overview) has examined the multiple cognitive approaches people use to solve arithmetic problems. A consensus in the field suggests that participants often deploy a wide range of strategies to solve arithmetic problems and that these strategies vary depending on the characteristics of the problem at hand, and the profile of the participant solving the problem. An understanding of the particular strategies used by particular participants is central to the study of human cognition (Gilmore, Göbel, \& Inglis, 2018) and has crucial practical implications related to helping people overcome learning deficits in mathematics (Geary \& Hoard, 2005). 
Three addend sums of the type $a+b+c$ offer a rich opportunity to directly observe the range of strategies that different participants may use because such sums afford the possibility of measuring a partial sum (i.e., $a+b$ or $a+c$ or $b+c)$. This partial sum, which participants are required to explicitly provide before entering the final sum, provides an objective indication of the particular strategy used by a participant - without relying on self-reports - which is an important goal for the psychologists researching mathematical cognition (Kirk \& Ashcraft, 2001; Metcalfe \& Campbell, 2011).

Strategies for three addend sums may, for example, include a preference to process addends in order in order to reduce the cognitive load associated with going out of order. Alternatively, participants may elect to switch orders to favor partial sums involving smaller numbers (e.g., for $9+2+3$, compute the partial sum $2+3$ ), partial sums that sum to 10 (e.g., for $6+2+8$, compute the partial sum $2+8$ ), or partial sums that feature the same digit (e.g., for $6+5+$ 6 , compute the partial sum $6+6)$. They therefore provide an attractive platform to investigate the particular types of strategies used by participants and the contexts in which they use them.

To get purchase on these issues, we began with an examination of prior findings related to multiple-strategy use in simple and complex arithmetic. We then sought to apply learnings from these contexts to develop hypotheses related to three addend addition. In particular we sought to examine two central questions: First, for what types of three addend problems do participants switch addend order? Second, does participant mathematical experience predict the contexts in which addend switching is more or less likely?

\section{Multiple Strategy Use in Simple Arithmetic}

In the context of simple arithmetic in general, and one-digit addition in particular, it is well understood that people use a range of different strategies. For example, a common strategy for simple one-digit addition is the retrieval of the answer from long-term memory (Geary \& Wiley, 1991). For example, when asked to add $6+7$ many people may recall that the accurate response without performing any computation. Alternatively, people use a derived strategy in which one sum is inferred from the recall of another. For example, if $6+6$ is recalled to be 12 , then $6+7$ is one more than 12 . Additionally, people may use a variety of decomposition strategies in which one or both addends are decomposed and recombined into smaller parts which are then added together. For example, $6+7$ may be decomposed as $3+3+7$ which is $3+10$.

Importantly, participant strategy may be influenced by a variety of factors related to the characteristics of problem at hand as well as factors related to the participant's profile. Factors related to the problem include operand order (e.g., participants may use a different strategy for $6+7$ than they do for $7+6$; Rickard, Healy, \& Bourne, 1994) and problem difficulty (e.g., participants may use different addition strategies for easier problems involving smaller addends such as $2+3$, than they do for more difficult problems with larger addends $9+7$; Zbrodoff, 1995).

Factors related to the participant's profile include a participant's mathematical experience and knowledge (Geary, Hoard, Byrd-Craven, \& DeSoto, 2004), intelligence (Geary, 2011), age (Lemaire, Arnaud, \& Lecacheur, 2004), and social-background (Bjorklund, Hubertz, \& Reubens, 2004).

\section{Multiple Strategy Use in Complex Arithmetic}

A growing body of evidence (see Cohen Kadosh \& Dowker, 2014, for an overview) suggests that, like simple arithmetic, computations involving complex arithmetic also feature multiple strategies whose deployment depends on factors related to the characteristics of the problem and the profile of the participant.

In particular, common strategies for multidigit addition may include direct retrieval (e.g., recalling that $12+47=$ 59), rounding the first and/or second operand up or down (e.g., $12+47$ may rounded as $10+50+2-3$ ), or columnar retrieval $(2+4$ and $2+7)$. Prior work has identified at least nine strategies that are used by children and adults (Lemaire \& Arnaud, 2008)

As in the case of simple arithmetic, participant strategy in complex arithmetic is known to depend on the characteristics of the problem at hand. Once again, a key contributor to strategy choice is known to be problem difficulty. In general, problem difficulty may vary with the size of the operands - the larger the operands the more difficult 
the problem (LeFevre, Sadesky, \& Bisanz, 1996). Other factors, such as the presence of a carryover are also known to contribute to problem difficulty in complex arithmetic (Lemaire \& Lecacheur, 2001)

Participant strategy in complex arithmetic is also known to be affected by age and basic arithmetic fluency (Lemaire $\&$ Brun, 2017). The extent and type of prior mathematical education are also known to play a role in strategy selection (e.g., Peters et al., 2013; Torbeyns et al., 2009).

\section{Applying Prior Findings to Three Addend Sums}

As discussed above, findings from both simple and complex arithmetic suggest that participants deploy a wide range of strategies that depend on problem characteristics as well as participant profiles. In this work, we sought to understand the range of strategies that people use for solving three addend sums. To achieve this goal, we presented participants with sums of different characteristics and examined if and when they added sums out of order. We reasoned that adding sums out of order involves increased cognitive effort relative to the more automatized and less effortful left to right processing of addends. It would therefore be undertaken only when doing so reduced processing difficulty.

Examining partial sums may not reveal the full breadth of all strategies used in three addend sums: for example, asking for a partial sum precludes information about decomposition strategies. Nevertheless, examining the pattern of preferred partial sums does provide an objective measure for a broad range of strategies in three addend sums.

Findings related to the impact of particular participant profiles in strategy selection suggests that participants with greater mathematical education tend to use a broader range of more efficient strategies. We therefore focused on measuring the impact of mathematical education on strategy choice. This single factor is not the only individual difference variable that may impact strategy choice; Nevertheless, we reasoned that it was likely to be an impactful variable - particularly in the context of the present studies that featured adult participants.

\section{Developing Hypotheses Related to Problem Characteristics}

Our central guiding assumption in developing hypotheses in the context of three addend sums was that in general, participants would seek less difficult computations that are associated with decreased cognitive effort and postpone or avoid more difficult computations that are associated with increased cognitive effort. Psychologists have long believed that humans (and other animals) select actions to minimize effort (Hull, 1943). More recent experiments have provided robust evidence that this "law of least effort" applies not only to physical effort, but also cognitive effort (Ballé, 2002; Kool, McGuire, Rosen, \& Botvinick, 2010).

Computations that rely on declarative or procedural knowledge are likely to be less cognitively effortful and therefore less difficult. Declarative knowledge is information that can be retrieved from memory without hesitation (Miller \& Hudson, 2007) and is known at a glance (e.g. $2+2=4$ ). Declarative knowledge is likely to have low cognitive costs when it is strengthened by repetition (Ivarie, 1986; Miller \& Heward, 1992). Procedural knowledge is the ability to follow a set of steps to solve a mathematical task (Bottge, 2001; Carnine, 1997; Goldman \& Hasselbring, 1997). Procedural knowledge is likely to have low cognitive costs when the steps they entail are simple and frequently repeated.

What addend pairs are likely to feature low difficulty based on declarative and procedural knowledge? We propose that low-difficulty pairs due declarative knowledge include those in which both addends are small ( $\leq 5$, e.g., $2+3$ ), as well as those in which both addends are equal (e.g., $8+8$ ). The 'small number' proposal is supported by the problem size effect according to which reaction times to problems of the type ' $a+b=$ ?' increase with the size of the problem's addends (Groen \& Parkman, 1972). The 'equal addends' proposal is supported by the that 'a $+\mathrm{a}$ ' is computationally similar to 'a times 2', which is frequently reinforced via multiplication tables. We further propose that low-difficulty pairs due to procedural knowledge include those in which one of the addends is 10 (e.g., $10+9$ ). The 'adding to 10' proposal relies on the base-10 nature of arithmetic and that the procedure for adding a single-digit number is akin to the highly automated procedure of counting.

We next illustrate how information about low-difficulty partial sums can be used to predict three addend addition strategies. For example, in the sum $3+9+2$, we hypothesize that people are likely to first add $3+2$ (a low difficulty partial sum per the 'small number' proposal) to obtain the partial sum of 5 and then add 9 to obtain 14. Similarly, in the sum $8+7+8$, we hypothesize that people are likely to first add $8+8$ (a low difficulty sum per the 'equal addends' 
proposal) to obtain the partial sum of 16 , and then add 7 to obtain 23 . Related to the 'sum to 10 proposal', for example, in the sum $9+7+1$, we hypothesize that people are likely to first add $9+1-$ since doing so yields a partial sum of 10 which then affords a low-difficulty addition with 7 to obtain 17. Finally, we also hypothesize that people may proactively seek to perform sums to make the partial sum equal to the third addend. For example, in the sum $7+5+$ 2 , we hypothesize that people are likely to add $5+2$ to obtain a partial sum of 7 , which then provides access to the low-difficulty $7+7$ (per the 'equal addends' proposal). These considerations lead us to the first of six hypotheses tested in the present work:

Hypothesis 1 - Preference for Low Difficulty Partials: In the context of three addend sums, adults are more likely to pursue out-of-order addition when doing so involves (a) adding two smaller (e.g., $\leq 5)$ numbers, (b) two equal numbers, (c) completing a partial sum that (later) requires adding a number to 10 , or (d) completing a partial sum that (later) requires summing two equal digits.

Next, we consider the consequences of people's tendency to postpone or avoid cognitive effort. Due to the problem size effect (Groen \& Parkman, 1972), sums involving larger addends (e.g., $9+8+4$ ) in the first two slots may feature greater difficulty than alternative partial sums. Since, per our proposal, people may tend to postpone or avoid completing tasks featuring higher difficulty, it is possible that such sums are more likely to be completed out of order than other sums. This leads to the second of six hypotheses tested in the present work:

Hypothesis 2 - Avoidance of High Difficulty Partials: In the context of three addend sums, adults are more likely to pursue out of order addition when both the first two addends are large numbers (i.e., $\geq 8$ ).

The cognitive effort-based reasoning also suggests that three addend sums in which there are no switching advantages and do not feature two large numbers in the first two slots should be less likely to be completed out of order than other sums. This is because the cognitive effort of adding out of order will not be mitigated by other gains related to reduced-difficulty. For example, consider the sum $6+3+8$. In such cases, related to Hypothesis 1 , there are no small number pairs, equal numbers, partials that add to 10 , or partials that add to a third number. Further, related to Hypothesis 2, the first two numbers are not large (and therefore not avoided). Here, there is no apparent benefit of going out of order but doing so would involve the cognitive load related to keeping out-of-order numbers in working memory. These considerations lead to the third of six hypotheses tested in the present work.

Hypothesis 3 - Low Rate of Switching due to Avoidable Cognitive Effort: In the context of three addend sums, adults are less likely to pursue out of order addition when there are no normative benefits for doing so (i.e., those described in Hypothesis 1 and 2). In this case, participants would generally seek to avoid the cognitive effort of adding sums out of order.

Table 1 illustrates and summarizes Hypotheses 1-3.

Table 1

Examples Illustrating Study Hypotheses 1-3 and Rationales

\begin{tabular}{lccl}
\hline Hypothesis & Illustrative Example & Hypothesized Partial Sum & Rationale \\
\hline 1a (small) & $5+9+2$ & $5+2$ & Small Numbers added first \\
1b (equal) & $8+5+8$ & $8+8$ & Equal numbers added first \\
$1 \mathrm{c}(10 \mathrm{~s})$ & $9+7+1$ & $9+1$ & Partial Sum to 10 \\
1d ( $3^{\text {rd }}$ num) & $9+5+4$ & $5+4$ & Partial Sum equals $3^{\text {rd }}$ Number \\
2 (large) & $8+9+3$ & $8+3$ or $9+3$ & Larger Sums Avoided \\
3 (control) & $6+3+8$ & $6+3$ & Increased cognitive effort of going out of order; No associated benefits \\
\hline
\end{tabular}

Are some people more sensitive to reduced-difficulty related benefits increased cognitive effort related costs? We next examine three additional hypotheses related to individual differences in three addend sums. 


\section{Developing Hypotheses Related to Participant's Mathematical Education}

How might adults vary in their use of three addend strategies based on their mathematical education? One possibility is that upon attaining adulthood, most adults have encountered so many instances of single-digit addition that they are at the performance ceiling for all such pairs, and there are no meaningful differences in levels of difficulty across adults. Another possibility - and the one we develop in the present work - is that differences in difficulty persist into adulthood and remain salient enough to result in meaningful differences in addition strategies for sums involving three addends.

In this work, we used the number of mathematics courses in high school and beyond as a proxy for the level of automaticity related to number facts. We reasoned that a participant who had completed courses in, for example, algebra, pre-calculus, and calculus, had - due to repetition - had developed a greater facility than a participant who had not completed these courses.

Consider a group with relatively higher levels of math education. We hypothesize many of the scenarios described in Hypothesis 1 are most likely to be salient for this 'high math education' group. They are thus more likely (relative to a group with low math education) to go out of order in contexts in which they can maximize their opportunity to tackle addend pairs that offer them decreased difficulty. In particular, this group is more likely to go out of order to add small addends (Hypothesis 1a), equal addends (Hypothesis 1b), or complete partial sums that requires adding a number to 10 (Hypothesis 1c), or complete partial sums that require summing two equal digits (Hypothesis 1d). These considerations lead to the fourth of six hypotheses tested in the present work.

Hypothesis 4 - Reduced Difficulty-Related Switching for Higher Math Education Individuals: In the context of three addend sums, 'high math education' adults are more likely to pursue out-of-order addition than 'low math education' adults when doing so involves adding two small numbers (s 5) two equal numbers, or completing a partial sum that (later) requires adding a number to 10 , or (later) requires summing two equal digits.

Conversely, the relative difficulty of summing two large numbers (c.f. Hypothesis 2) is likely to be higher for 'low math education' participants. Our fifth hypothesis is that these participants are more likely to pursue out of order sums to avoid adding two larger digits.

Hypothesis 5 - Greater Switching to Avoid Large Number Sums in Lower Math Education Individuals: In the context of three addend sums, 'low math education' adults are more likely to pursue out-of-order addition than 'high math education' adults when doing so involves sums in which both the first two addends are large numbers (e.g., $\geq 8$ ).

Finally, consider the case of numbers that did not offer any switching advantages and do not feature two large numbers in the first two slots (c.f. Hypothesis 3). We propose that low math education participants are more likely to switch for such sums than high math education participants. This is because lower levels of knowledge about number facts may often cause individuals in the low math group to be more likely to rely on backup math strategies such as verbal counting (Geary \& Wiley, 1991; Ostad, 1997), possibly even as adults. Verbal counting is initiated when the activation related to the counting process exceeds the activation related to the memory of the answer. Here, the participant may count on from the larger addend by the smaller addend relying on the 'min' strategy (Groen \& Parkman, 1972). For example, while adding $6+9$ individuals may start with the larger number 9 and then count up by 6 . An adult relying on min-based verbal counting strategies an adult is more likely to go out of order because they will start with the largest addend and add the smallest available addend to that. For example, in the sum $5+9+2$, a participant may start with 9 , count up by 2 , and then add 5 . This pattern of addition strategy would cause low math education adults to switch out of order in sums where there are no normative advantages related to switching. This leads to the final of six hypotheses tested in the present work.

Hypothesis 6 - Greater Switching to Avoid Large Number Sums in Lower Math Education Individuals:

'Low math education' adults are more likely to pursue out-of-order addition than 'high math 
education' adults in sums in which there are no normative advantages related to switching and the first two numbers are not large.

Table 2 illustrates and summarizes Hypotheses 4-6.

Table 2

Examples Illustrating Study Hypotheses 4-6 and Rationales

\begin{tabular}{lccl}
\hline Hypothesis & Illustrative Example & Participants More Likely to Go & \\
Out of Order & Rationale \\
\hline $4 \mathrm{a}($ small $)$ & $5+9+2$ & Higher Math Education & Reduce Difficulty \\
$4 \mathrm{~b}($ equal $)$ & $8+5+8$ & Higher Math Education & Reduce Difficulty \\
$4 \mathrm{c}(10 \mathrm{~s})$ & $9+7+1$ & Higher Math Education & Reduce Difficulty \\
$4 \mathrm{~d}\left(3^{\text {rd }}\right.$ num $)$ & $9+5+4$ & Higher Math Education & Reduce Difficulty \\
5 (large) & $8+9+3$ & Lower Math Education & Avoid Difficult Partials \\
6 (control) & $6+3+8$ & Lower Math Education & No switching advantages. \\
& & & Back-up Strategies Used \\
\hline
\end{tabular}

We tested the above hypotheses in two studies described below. In Study 1, we asked a sample of Internet workers of varying math education levels to solve 120 three-addend sums with different problem characteristics. This study allowed us to test each of the six hypotheses discussed above. We will note that at the outset of this study, it was not clear to us that adults - many of whom would have encountered thousands of instances of single-digit addition would meaningfully vary in experienced difficulty levels related to additions and whether such variances would lead to detectable differences in computation strategies. In Study 2 we re-used the same problem sets from Study 1 in a laboratory setting. This afforded the opportunity to confirm that participants in-fact used the strategies that we inferred from their partial sums in Study 1. In Study 2, we also measured response latencies for each question type.

\section{Study 1: Profiling Strategies in Three Addend Sums}

In Study 1 we asked participants to complete three addend sums by first calculating a partial sum. Our goal was to test each of the six hypotheses described above.

\section{Method}

We recruited 159 adult participants (median age range $=35-40$ ) through Amazon's Mechanical Turk crowdsourcing platform. Participants were informed that they would be asked to complete unique single-digit addition problems with three integers. They were told that they were "free to add the three numbers in whatever order [they] prefer." Participants were then given a sample problem $(6+5+3)$ and were shown that several partial sums $(11,9,8)$ were possible depending on the order they chose to sum the addends. They were again reminded that the choice of the order was up to them.

Participants were walked through four example problems in which they were to add three integers in varying orders. For both the training phase and main task, participants were instructed to report both the partial sum of the first pair of integers they chose to add and the total sum of all three integers. For example, a potential partial sum of the problem: $9+7+1$ is 10 and the total sum is 17 . Participants were instructed to record their partial sum as a numeric value in a designated labeled text box. They were then instructed to record the total sum of all three integers in a separate but adjacently labeled text box. An example of a problem trial can be seen in Figure 1. 
Figure 1

An Example Problem (1 out of 120) Seen by a Participant

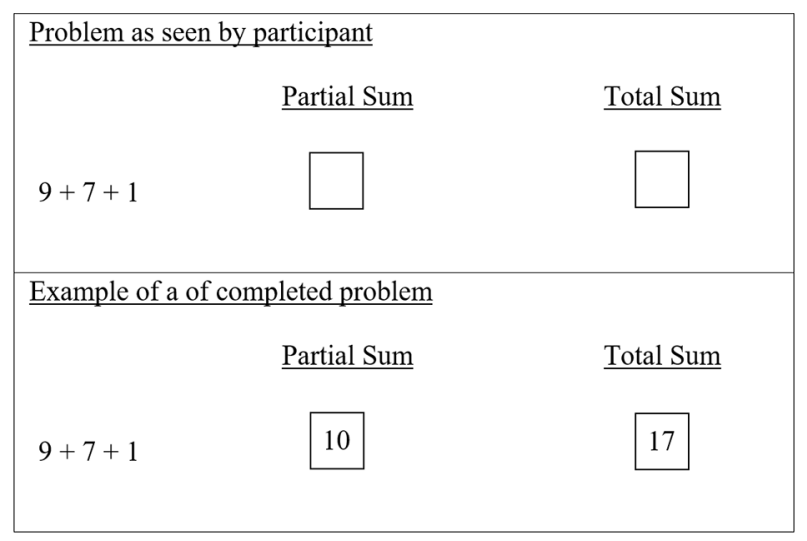

Note. In the illustrative example in the lower panel, the participant provided a partial sum of 10 (i.e. they added $9+7+1$ out of order). Alternatively, they could have provided partial sums of 16 (staying in order) or 8.

Participants were permitted to use pen and paper and asked to complete - in order - the 120 problems in one sitting without taking a break. All participants were given the same 120 problems, but the order of their presentation was randomized for each participant. Responses for the final sum of each problem were verified to be accurate in real-time. A notification would alert the participant if an incorrect total sum had been entered and they were prevented from continuing until they provided the correct answer.

The 120 questions belonged to 6 groups, each consisting of 20 questions. A summary of the types of questions in each group can be seen in Table 3. Problems in each group, excluding problems in the control group, contained two integers that would add to a specific partial sum positioned at either the first and third or second and third integer locations. Problems were divided equally among these two locations.

Table 3

Group Structures

\begin{tabular}{lcccc}
\hline & & & \multicolumn{2}{c}{ Integers Adding to Specific Partial Sum } \\
\cline { 3 - 5 } Group & Description & Hypothesis & First and Third & Second and Third \\
\hline A & Equal numbers & $1 \mathrm{~b}$ & $\mathbf{6 + 3 + 6}$ & $4+\mathbf{9}+\mathbf{9}$ \\
$\mathrm{B}$ & Partial sum to 10 & $1 \mathrm{c}$ & $\mathbf{4 + 7 + 6}$ & $9+\mathbf{6}+\mathbf{8}$ \\
$\mathrm{C}$ & Partial sum equals 3 ${ }^{\text {rd }}$ addend & $1 \mathrm{~d}$ & $\mathbf{7 + 9 + 2}$ & $8+\mathbf{3}+\mathbf{5}$ \\
$\mathrm{D}$ & Partial sum to 12 & Exploratory & $\mathbf{3}+8+\mathbf{9}$ & $5+\mathbf{4}+\mathbf{8}$ \\
$\mathrm{E}$ & Partial sum to a multiple of 5 & Exploratory & $\mathbf{8}+6+\mathbf{7}$ & $7+\mathbf{4}+\mathbf{1}$ \\
$\mathrm{F}$ & Control & 3 & $6+3+8$ & $2+9+4$ \\
\hline
\end{tabular}

Group A (equal numbers) contained two equal integers of the same value in each problem. Group B (partial sum to 10) contained two integers that created a partial sum of 10. Group $C$ (partial sum to $3^{\text {rd }}$ addend) consisted of problems including two integers, that when added, would equal the value of the third remaining integer. Group D (partial sum to 12) was the first of two exploratory groups where two integers would create a partial sum to 12 . We included this group since a duodecimal or base 12 system commonly occurs in daily mathematical interactions with clocks, calendars, and measurements (imperial feet and inches). Group E (partial sum to multiple of 5), the second of the two exploratory groups contained two integers that created a partial sum to an integer multiple of 5 (excluding the value 10). We included this partial sum as an exploratory group because counting by 5 's is often taught to children counting on their 
hands and feet. The final group was termed a control group as it consisted of randomly generated numbers under the condition that the numbers chosen did not fit the partial sum characteristics of the five previously mentioned groups.

The 120 problems were generated by software that was constrained by the requirements of each condition. For each group and each problem, the program initially randomly produced 3 ordered single-digit numbers between 1 through 9 (inclusively) for a problem. The software then checked that the problem met the partial sum requirements of the group it belonged to, did not simultaneously satisfy the requirements of a different group, and had not already been included in the problem list.

If any of the requirements were not met, the three numbers were discarded, and the program generated a new random set of 3 integers. This process was iterated until 20 problems had been generated for each respective group. For each group, 10 sets of questions were generated with the first and third integers of the problem adding up to the specific partial sum of its respective group, and 10 sets of questions were generated with the second and third integers of the problem adding up to the specific partial sum of its respective group.

After they completed all one-hundred-twenty problems, participants were asked to report their prior experience with mathematics (see Supplementary Materials). We first asked participants about their highest level of math education through a multiple-choice response: (1) I have not taken algebra and geometry courses (2) I have taken algebra and geometry courses (3) I have had calculus but not more advanced courses (4) I have had more advanced math courses but do not have a STEM background (5) I have a STEM or technical degree. These distinctions between math education levels were drawn through our analysis of common core curricula in the United States. We recognize this is an imperfect measure since the correlation between more math courses and number automaticity is not perfect. Nevertheless, we reasoned that it was the most objective measure available to us.

\section{Results and Discussion}

Overall, Hypotheses $1-3$ were fully supported. Hypothesis 4 was partially supported, Hypothesis 5 was not supported, and Hypothesis 6 was fully supported. We examine each result in turn.

Hypothesis 1a was related to the greater propensity among all adults to switch the order of addition when either the first and third addend were small and the second addend was large (e.g., $4+9+3$ ), or when the first addend was large and the second and third addend were small (e.g., $9+4+3$ ). In either case, we expected such sums to be completed out of order at greater rates than the out of order rates in the control group. This was indeed the case. The switching rate for the control group was $38.9 \%$. When the large addend was specified as a number greater than or equal to 8 , and the small addends were specified as numbers $\leq 5$, the overall switching rate was $52.27 \%$. The difference in switching rates is significant $\chi^{2}(1,9989)=46.18, p<.001$. Similar switching rates were found for equivalent specifications of large and small numbers. For example, in the case large numbers $=9$ and small numbers $\leq 3$, the switching rate was $49.06 \%$. However, switching rates for cases in which the large number $=7$ and the small numbers $\leq 5$ were not statistically different from the control group, suggesting potential processing difference for large addends $\geq 8$. Switching rates for each sum are included in the Supplementary Materials.

We next confirmed Hypothesis $1 \mathrm{~b}$ - 1d (i.e., higher switching rates for equal numbers, partial sums involving adding a number to 10, and partial sums involving two equal numbers). Hypothesis 2 related to the greater propensity among all adults to switch the order of addition when both the first and second addend were large $(9+8+3)$ was confirmed. Further, Hypothesis 3 related to reduced switching for Control trials relative to all other trials was also confirmed. The results are shown in Table 4.

Neither the exploratory 'sum to 12 ' group (in which an out of order partial sum added to 12), nor the exploratory 'Sum to Multiple of 5 ' group were associated with an increased probability of switching relative to control trials. 
Table 4

Study 1 Switching Rates by Class of Problem (Compared to Control)

\begin{tabular}{lcc}
\hline Group & Switch Rate $\%$ & $\chi^{2}$ \\
\hline Control Group & $\mathbf{3 8 . 9 0}$ & - \\
Small Numbers (H1a: $4+9+3)$ & 52.27 & $124.12^{* * *}$ \\
Equal Addends (H1b: $8+5+8)$ & 49.06 & $66.58^{* * *}$ \\
Sums to Ten (H1c: $9+7+1)$ & 50.09 & $80.69^{* * *}$ \\
Sums to Third Addend (H1d: $9+4+5)$ & $49.06^{\text {a }}$ & $66.58^{* * *}$ \\
Large Addends (H2: $9+8+3)$ & 45.80 & $16.37^{* *}$ \\
Control < Sample (H3) & $43.58^{\text {b }}$ & $34.07^{* * *}$ \\
\hline
\end{tabular}

${ }^{\mathrm{a} I d e n t i c a l ~ r a t e ~ w i t h ~ H 1 b ~ i s ~ c o i n c i d e n t a l . ~}{ }^{\mathrm{b}} \mathrm{S} w i t c h$ Rate for Non-Control Addends.

${ }^{*} p<.05 .{ }^{* *} p<.01 .{ }^{* * *} p<.001$.

Hypothesis 4-6 involved examining the consequences of (predicted) differences in levels of math education across adults. We predicted that a higher number of years of mathematics education would result in an increased proclivity to go out of order to sum small addends, equal addends, create a partial sum of 10, and to create a partial sum equal to the third number (Hypothesis 4). As shown in Table 5, Hypothesis 4, tested via a logistic regression (since we sought to predict the occurrence of a switch vs. no-switch), was partially supported so that the tendency to go out of order increased with the level of math education (for small addends and for equal addends).

Table 5

Summary of Regression Analysis for Interaction Between Type of Problem and Education Level (Hypotheses 4a-d, 5, 6)

\begin{tabular}{lcc}
\hline Group & Estimate & Standard Error \\
\hline Small Addends (H4a) & $0.07^{* *}$ & 0.02 \\
Equal Addends (H4b) & $0.17^{* * *}$ & 0.03 \\
Sums to Ten (H4c) & -0.01 & 0.03 \\
Sums to Third Addend (H4d) & 0.00 & 0.03 \\
Large Addends (H5) & 0.05 & 0.05 \\
Control $(6+3+8)$ & $-0.09^{* *}$ & 0.03 \\
${ }^{*} p<.05^{* *} p<.01^{* * *} p<.001$ & &
\end{tabular}

${ }^{*} p<.05 .{ }^{* *} p<.01 .{ }^{* * *} p<.001$.

We further predicted that lower mathematics education would result in an increased proclivity to switch the order of sums in which the first two addends were large and in control sums. As shown in Table 5, Hypothesis 5 was not supported (lesser math education did not predict more switching for sums in which the first two addends were large). Hypothesis 6 was supported (lesser math education did not predict more switching for sums in which the first two addends were large).

Overall Hypotheses 4-6 suggested that higher math education was related to switching to leverage efficient strategies (e.g., double sums were switched at greater rates; see Figure 2) and lower math education was related to switching without identifiable gains in efficiency (e.g., switching for control sums). 
Figure 2

Probability of Switching by Math Education Level for Different Problem Types

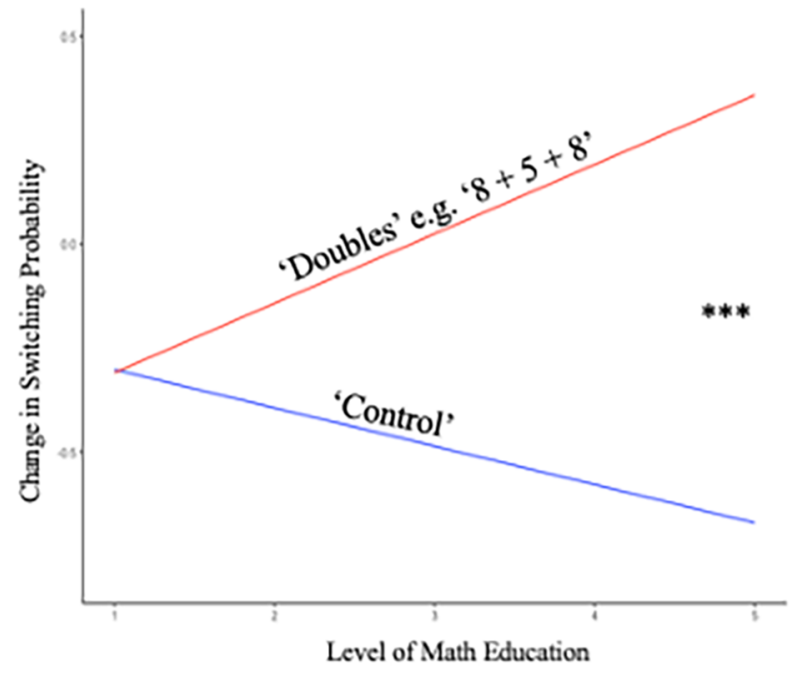

Note. More mathematics courses were generally associated with increased switching for double sums where prior facility with adding the same number to itself could be leveraged. Conversely, fewer mathematics courses were generally associated with increased switching for control sums that did not feature any partial sums with normative benefits to switching.

Finally, we were intrigued by why people with lower levels of math education were going out of order in Control sums - for which there no normative benefits of switching. We explored the possibility that in the absence of the ability to readily recall sums, people may tend to compute a partial sum by starting with the largest number and adding the smallest number to it. For example, in the $6+3+8$ problem, they might start with 8 and add 3 to get a partial sum of 11. We did not find supporting evidence for this 'add from largest' strategy since partial sums of the type max $+\min$ occurred at the same (statistical) rate as other partial sums in the 'low education' group.

Study 1 was an important first step in examining how participants solve three single-digit addend problems. However, it did not provide response latency data that is crucial to establish the relative strategy benefits of each type of strategy. Study 2 addressed this limitation.

\section{Study 2: Measuring Response Latencies With an In-Person Study}

Study 2 had three goals: First, we sought to replicate a portion of the results in Study 1 using an in-person study. We reasoned that an in-person study could validate the pattern of results obtained via online methods. Second, we sought to examine whether response latency data, measurable in an in-person study, was consistent with our assumptions related to problem difficulty and cognitive effort. We expected that response latency times for 'equal addends', 'sum to 10', and 'sum to third addend' would be less than response latency times for the control group. Third, we sought to use verbal reports to obtain participant justifications for their chosen strategy.

\section{Method}

Informed by the effect-sizes in Study 1, we recruited 50 community volunteers (22 females) with a mean age of 24.5 years. Participants were asked to complete an identical set of problems to those participants in Study 1. However, Study 2 was conducted in-person. Unlike in Study 1, there were no meaningful differences in the prior math experience of the participant sample (all had completed at least one college level math course). We therefore exclusively focused on strategy differences due to problem characteristics - and not due to differences in math education. 
The participant instructions and protocols for Study 2 were similar to those in Study 1 with two key differences. First, after entering a partial sum, participants were required to press the 'tab' key, which made an additional text field appear - in which they then entered their final response. This alteration allowed us to measure participant response latencies for partial sums as well as response latencies for final sums. Second, after completing 120 sums, participants were asked to provide verbal reports of their strategy selection process for eight problems in which they switched the addition order - two each from Group A (equal addends), Group B (Partial sum to 10), Groups C (Partial sum equals 3rd number), and Group F (Control). In these questions, participants were provided a report of the partial sum they obtained, and then asked to provide an explanation of why they picked their strategy via writing 1-2 sentences in an open response format.

An unusual feature of the study was that due to concerns related to the COVID-19 pandemic, participants were asked to wear a mask and surgical gloves for the duration of the study. It is possible that this slightly increased the response latencies we recorded.

\section{Results and Discussion}

Overall, the results of Study 2 closely resembled the results of Study 1 - thereby providing an in-person replication of an online study. Further, patterns of response latencies supported assumptions related to problem difficulty and cognitive effort. Finally, participant explanations for their strategy choices were in-line with hypothesized justifications for factors contributing to switching.

Replicating Study 1, the switching rates for 'equal addends', 'sum to 10', and 'sum to third addend' problem types were significantly higher than for control problems (Table 6).

Table 6

Study 2 Switching Rates by Class of Problem (Compared to Control)

\begin{tabular}{lcc}
\hline Group & Switch Rate $\%$ & $\chi^{2}$ \\
\hline Control Group & 36.57 & - \\
Small Numbers $(4+9+3)$ & 53.35 & $58.53^{* * *}$ \\
Equal Addends $(8+5+8)$ & 53.12 & $53.09^{* * *}$ \\
Sums to Ten $(9+7+1)$ & 51.83 & $45.72^{* * *}$ \\
Sums to Third Addend $(9+4+5)$ & 51.08 & $41.17^{* * *}$ \\
Large Addends $(9+8+3)$ & 51.54 & $22.54^{* * *}$ \\
Control $<$ Sample & 30.03 & $34.07^{* * *}$ \\
${ }^{*} p<.05 .{ }^{* *} p<.01 .{ }^{* * *} p<.001$. & &
\end{tabular}

We had hypothesized that the total response latencies of problems in the 'equal addends', 'sum to 10', and 'sum to third addend' groups would be less than that of the overall response latency of problem types in the Control group. This was found to be the case.

The response latency across all problems was 6.23 seconds with a standard deviation of 3.34 seconds. We removed 212 outliers (out of 6000 responses) with response latencies greater than 20 seconds ( +4 standard deviations from the mean). The response latency for problems in the control group was 6.61 seconds; for problems in the 'equal addends' group it was 6.13 seconds, $t(1918)=-3.05, p=.002$; for problems in the 'sum to 10 ' group it was 5.70 seconds, $t(1934)=$ $-6.14, p<.001$; and for problems in the 'sum to third' group, it was 5.99 seconds, $t(1924)=-4.02, p<.001$. These findings are consistent with our assumption that there were efficiencies related to switching the order of addends in these types of questions.

We split the latency times into the pre- and post- partial sum intervals. The results are shown in Figure 3 below. 
Figure 3

Response Latency Data for Different Problem Types

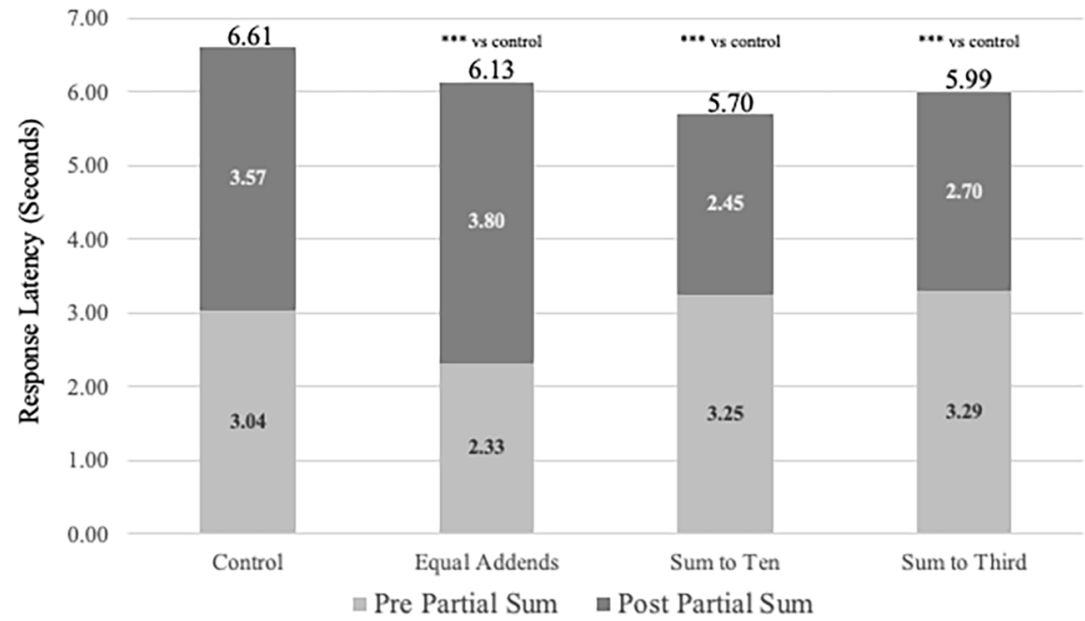

Note. Compared to the control group, other problem types feature high rates of switching had reduced latencies. Pre- and post- partial sum splits supported hypothesized sources of efficiency. For example, the 'equal addends' problem type (e.g., $8+5+8)$ had a relatively small pre-partial latency, presumably due to efficiency related adding a number to itself (e.g., $8+8$ ). Conversely the 'sum to 10 ' problem type (e.g., $9+7+1)$ had a relatively small post-partial sum latency, presumably due to efficiencies related to adding a number to 10. (e.g., $10+7$ )

Notably, the lower average response latency of the 'Equal Addends' problem type was exclusively due to a reduced latency in the pre partial sum interval. This is consistent with our assumption of greater facility related to adding a number to itself. For example, in computing the sum $8+5+8$, we would predict that $8+8$ has low response latency and that is what we observed.

Contrastingly, the lower average response latency of the 'Sum to Ten' problem type was exclusively due to a reduced latency in the post partial sum interval. This is consistent with our assumption of greater facility related to adding any number to ten. For example, in computing the sum $8+7+2$, we would predict that $10+7-$ a computation that occurs after the partial sum of $8+2$ has been computed will have response latency - and that is what we observed. A similar pattern was seen in 'Sum to Third' problem type where the post partial sum interval was exclusively responsible for the reduced average response latency compared to the control group. This is consistent with our assumption of greater facility related to adding a partial sum to itself. For example, in adding $9+4+5$, the efficiencies appear to occur after $4+$ 5 have been added to obtain a partial sum of 9 - which can then quickly be added to itself.

Finally, written participant explanations of why they switched the order of addends, coded by an experimenter who was blind to the condition, were almost always of three main types: Either participants reported that they did not know their reasons for switching, or they reported an idiosyncratic reason (e.g., "I have always liked the Number 6."), or they identified the expected justification for the particular problem type (e.g., "I wanted the numbers to sum to ten."). The explanation breakdown for each problem type was as follows (the \% may not sum to 100 because of a minority of responses being classified into an 'other' category): 'Equal Addends' - Don't know 4\%, Idiosyncratic 15\%, Expected 72\%; 'Sum to 10 ' - Don't know 4\%, Idiosyncratic 5\%, Expected 84\%; 'Sum to Third' - Don't know 31\%, Idiosyncratic $12 \%$, Expected 55\%; 'Control' - Don't know 41\%, Idiosyncratic 48\%.

\section{General Discussion}

Prior research has demonstrated that computations involving simple and complex arithmetic feature multiple strategies whose deployment depends on factors related to the characteristics of the problem and the profile of the participant. In this work we made progress towards understanding how these factors influence the computation of single-digit, three-addend sums. These sums offer the advantage that they often require the computation of a partial sum prior to 
the computation of the final answer, and the particular partial sum chosen is often indicative of the underlying strategy implemented by the participant. Thus, examining mathematical strategies in the context of three-addend sums have the potential to reduce reliance on self-report

We hypothesized that participants would switch the order of addition to prioritize addends that were small $(5+9+$ 3 ), or equal $(8+5+8)$ or when doing so led to a partial sum of $10(9+7+1)$, or a partial sum that was equal to the remaining third addend $(9+4+5)$. Each of these hypotheses was confirmed. We also hypothesized that participants would prefer going out of order if doing so would avoid or postpone adding sums with large addends $(8+9+3)$ since they required greater effort, sooner. Our results supported this hypothesis. We also predicted that people would be less likely to go out of order when doing so did not offer efficiency gains. Consistent with this hypothesis, participants' switching rates for problems in the control group was lower than for problems in other groups. These results were confirmed both via an on-line and an in-person study. Response latency data in the in-person study data demonstrated that the efficiency gains in these problems were predictably either before the partial sum (e.g., when equal addends were present) or were after the partial sum (e.g., when a partial sum of 10 was computed).

Related to individual differences, consistent with our hypotheses, we found that participants with greater mathematical experience were more likely (compared to participants with lesser mathematical experience) to go out of order to exploit efficiencies related to adding relatively small numbers and adding equal numbers. Further, as hypothesized, individuals with lesser mathematical experience were more likely (compared to participants with greater mathematical experience) to go out of order in problems in the control group.

Notably, our results did not support our hypotheses that people with greater mathematical background were more likely (relative to people with lesser mathematical experience) to approach problems in which a partial sum led to 10 or in which a partial sum led to a number equal to the remaining third addend - even though across all participants people did prefer to switch in such sums. This is consistent with prior observation that indicates that computational skill and problem-solving may be separable aspects of mathematical cognition (Fuchs et al., 2008). Future work is required to test this possibility and determine its boundary conditions.

Our results also did not support the hypothesis that people with greater mathematical background were less likely (relative to people with lesser mathematical experience) to avoid problems that featured two large addends. At first appraisal, the lack of an interaction for such problems suggests that there is no meaningful difference in the automaticity levels of adding large addends for participants with greater or lesser mathematical experience. An alternative reappraisal is that it is not the absolute level of automaticity that determines choice but the relative level of automaticity. The partial sum of $9+8$ may indeed have greater absolute levels of automaticity for people with greater levels of mathematical experience than for people with lower mathematical experience, but the relative difference in automaticity levels between the different participants may be relatively constant. It is, therefore, possible that all participants switched away from large addends at equivalent rates because relative to other available partial sums, these addends offered relatively lower automaticity. We acknowledge that this explanation is post hoc and requires rigorous empirical testing.

Our results did support our hypothesis that participants with lower levels of mathematical experience are more likely to switch away from control problems in which there were no normative automaticity advantages. We had predicated this hypothesis on the assumption that participants with lower automaticity may use the min addition strategy (Siegler \& Jenkins, 2014) that involves counting up from a large number. We had reasoned that people with low automaticity would resort to the min strategy since they could not rely on recalling each of the relevant partial sums from memory. However, we found no evidence of increased min strategy usage in participants with lower mathematical experience. This leaves open the intriguing question as to why participants with low levels of mathematical experience tended to switch the addition order for control problems in which there was no apparent normative benefit of doing so - even though adding out of order entails increased cognitive load.

We close this work with the observation that while the study of three addend sums is firmly rooted in the mathematical cognition literature, it has the potential to open a new domain for research into value-based decision making (Rangel, Camerer, \& Montague, 2008; Suri, Shine, \& Gross, 2018) and in other domains. Researchers in several sub-disciplines study contexts in which decision-making process unfolds via a comparison of the costs and benefits on offer. All else being equal, people seek to embrace options with higher benefits and avoid options with higher costs. In 
the context of three-addend sums the benefit of efficient summing (e.g., adding a number to 10) must be compared with the cognitive cost of processing addends out of order. When and for whom do the benefits exceed the costs? Continued research addressing this question has the potential to generatively inform multiple literatures in psychology.

Funding: The authors have no funding to report.

Acknowledgments: The authors have no additional (i.e., non-financial) support to report.

Competing Interests: The authors have declared that no competing interests exist.

\section{Supplementary Materials}

The Supplementary Materials contain a list of all three number addends and their switching rates (for access see Index of Supplementary Materials below).

\section{Index of Supplementary Materials}

Sharma, M., Bashir, S., \& Suri, G. (2021). Supplementary materials to "Three addend addition: Who goes out of order and why?" [Additional information]. PsychOpen GOLD. https://doi.org/10.23668/psycharchives.4706

\section{References}

Ballé, M. (2002). La loi du moindre effort mental [The law of less mental effort]. Sciences Humaines, 128, 36-39.

Bjorklund, D. F., Hubertz, M. J., \& Reubens, A. C. (2004). Young children's arithmetic strategies in social context: How parents contribute to children's strategy development while playing games. International fournal of Behavioral Development, $28,347-357$. https://doi.org/10.1080/01650250444000027

Bottge, B. A. (2001). Reconceptualizing mathematics problem solving for low-achieving students. Remedial and Special Education, 22(2), 102-112. https://doi.org/10.1177/074193250102200204

Campbell, J. I. (Ed.). (2005). Handbook of mathematical cognition. New York, NY, USA: Psychology Press.

Carnine, D. (1997). Instructional design in mathematics for students with learning disabilities. fournal of Learning Disabilities, 30(2), 130-141. https://doi.org/10.1177/002221949703000201

Cohen Kadosh, R., \& Dowker, A. (Eds.). (2014). Oxford handbook of numerical cognition. Oxford, United Kingdom: Oxford University Press.

Fuchs, L. S., Fuchs, D., Stuebing, K., Fletcher, J. M., Hamlett, C. L., \& Lambert, W. (2008). Problem solving and computational skill: Are they shared or distinct aspects of mathematical cognition? Journal of Educational Psychology, 100, 30-47.

https://doi.org/10.1037/0022-0663.100.1.30

Geary, D. C. (2011). Cognitive predictors of achievement growth in mathematics: A 5-year longitudinal study. Developmental Psychology, 47, 1539-1552. https://doi.org/10.1037/a0025510

Geary, D. C., \& Hoard, M. K. (2005). Learning disabilities in arithmetic and mathematics: Theoretical and empirical perspectives. In J. I. D. Campbell (Ed.), Handbook of mathematical cognition (pp. 253-267). New York, NY, USA: Psychology Press.

Geary, D. C., Hoard, M. K., Byrd-Craven, J., \& DeSoto, M. C. (2004). Strategy choices in simple and complex addition: Contributions of working memory and counting knowledge for children with mathematical disability. fournal of Experimental Child Psychology, 88, 121-151. https://doi.org/10.1016/j.jecp.2004.03.002

Geary, D. C., \& Wiley, J. G. (1991). Cognitive addition: Strategy choice and speed-of-processing differences in young and elderly adults. Psychology and Aging, 6, 474-483. https://doi.org/10.1037/0882-7974.6.3.474

Gilmore, C., Göbel, S. M., \& Inglis, M. (2018). An introduction to mathematical cognition. Abingdon, United Kingdom: Routledge.

Goldman, S. R., \& Hasselbring, T. S. (1997). Achieving meaningful mathematics literacy for students with learning disabilities. fournal of Learning Disabilities, 30, 198-208. https://doi.org/10.1177/002221949703000207 
Groen, G. J., \& Parkman, J. M. (1972). A chronometric analysis of simple addition. Psychological Review, 79, 329-343. https://doi.org/10.1037/h0032950

Hull, C. L. (1943). Principles of behavior (Vol. 422). New York, NY, USA: Appleton-Century-Crofts.

Ivarie, J. J. (1986). Effects of proficiency rates on later performance of a recall and writing behavior. Remedial and Special Education, 7 , 25-30. https://doi.org/10.1177/074193258600700506

Kirk, E. P., \& Ashcraft, M. H. (2001). Telling stories: The perils and promise of using verbal reports to study math strategies. fournal of Experimental Psychology: Learning, Memory, and Cognition, 27, 157-175. https://doi.org/10.1037/0278-7393.27.1.157

Kool, W., McGuire, J. T., Rosen, Z. B., \& Botvinick, M. M. (2010). Decision making and the avoidance of cognitive demand. Fournal of Experimental Psychology: General, 139, 665-682. https://doi.org/10.1037/a0020198

LeFevre, J.-A., Sadesky, G. S., \& Bisanz, J. (1996). Selection of procedures in mental addition: Reassessing the problem size effect in adults. Fournal of Experimental Psychology: Learning, Memory, and Cognition, 22, 216-230.

https://doi.org/10.1037/0278-7393.22.1.216

Lemaire, P., \& Arnaud, L. (2008). Young and older adults' strategies in complex arithmetic. The American fournal of Psychology, 121, 1-16. https://doi.org/10.2307/20445440

Lemaire, P., Arnaud, L., \& Lecacheur, M. (2004). Adults' age-related differences in adaptivity of strategy choices: Evidence from computational estimation. Psychology and Aging, 19, 467-481. https://doi.org/10.1037/0882-7974.19.3.467

Lemaire, P., \& Brun, F. (2017). Age-related changes in children's strategies for solving two-digit addition problems. fournal of Numerical Cognition, 3, 582-597. https://doi.org/10.5964/jnc.v3i3.117

Lemaire, P., \& Lecacheur, M. (2001). Older and younger adults' strategy use and execution in currency conversion tasks: Insights from French franc to euro and euro to French franc conversions. Fournal of Experimental Psychology: Applied, 7, 195-206. https://doi.org/10.1037/1076-898X.7.3.195

Metcalfe, A. W., \& Campbell, J. I. (2011). Adults' strategies for simple addition and multiplication: Verbal self-reports and the operand recognition paradigm. fournal of Experimental Psychology: Learning, Memory, and Cognition, 37, 661-672. https://doi.org/10.1037/a0022218

Miller, A. D., \& Heward, W. L. (1992). Do your students really know their math facts? Using daily time trials to build fluency. Intervention in School and Clinic, 28, 98-104. https://doi.org/10.1177/105345129202800205

Miller, S. P., \& Hudson, P. J. (2007). Using evidence-based practices to build mathematics competence related to conceptual, procedural, and declarative knowledge. Learning Disabilities Research \& Practice, 22, 47-57. https://doi.org/10.1111/j.1540-5826.2007.00230.x

Ostad, S. A. (1997). Developmental differences in addition strategies: A comparison of mathematically disabled and mathematically normal children. The British fournal of Educational Psychology, 67, 345-357. https://doi.org/10.1111/j.2044-8279.1997.tb01249.x

Peters, G., De Smedt, B., Torbeyns, J., Ghesquière, P., \& Verschaffel, L. (2013). Children's use of addition to solve two-digit subtraction problems. British fournal of Psychology, 104, 495-511.

Rangel, A., Camerer, C., \& Montague, P. R. (2008). A framework for studying the neurobiology of value-based decision making. Nature Reviews Neuroscience, 9, 545-556. https://doi.org/10.1038/nrn2357

Rickard, T. C., Healy, A. F., \& Bourne, L. E. (1994). On the cognitive structure of basic arithmetic skills: Operation, order, and symbol transfer effects. Journal of Experimental Psychology: Learning, Memory, and Cognition, 20, 1139-1153.

https://doi.org/10.1037/0278-7393.20.5.1139

Siegler, R., \& Jenkins, E. A. (2014). How children discover new strategies. New York, NY, USA: Psychology Press.

Suri, G., Shine, J. M., \& Gross, J. J. (2018). Why do we do what we do? The attention-readiness-motivation framework. Social and Personality Psychology Compass, 12, Article e12382. https://doi.org/10.1111/spc3.12382

Torbeyns, J., De Smedt, B., Ghesquière, P., \& Verschaffel, L. (2009). Acquisition and use of shortcut strategies by traditionally schooled children. Educational Studies in Mathematics, 71, 1-17. https://doi.org/10.1007/s10649-008-9155-z

Zbrodoff, N. J. (1995). Why is $9+7$ harder than $2+3$ ? Strength and interference as explanations of the problem-size effect. Memory \& Cognition, 23, 689-700. https://doi.org/10.3758/BF03200922 


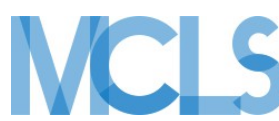

Journal of Numerical Cognition (JNC) is an official journal of the Mathematical Cognition and Learning Society (MCLS).
(P) leibniz-psychology.org

PsychOpen GOLD is a publishing service by Leibniz Institute for Psychology (ZPID), Germany. 